\title{
Obtained Carbon Nano-onions from Underwater Arc Discharge Without the Complex Purification Procedures
}

\author{
Salih Mohamed Osman 1, 3, *, Asma Mohamed Elhussein ${ }^{2}$, Fatma Osman Mahmoud ${ }^{1}$, \\ Mohammed Bilal Sabahelkher ${ }^{1}$, Lorenzo Caputi ${ }^{3}$, Andrea Scarcello ${ }^{3}$, Francesca Alessandro ${ }^{3}$ \\ ${ }^{1}$ Department of Physics, University of Kassala, Kassala, Sudan \\ ${ }^{2}$ Qilwah College of Science and Arts, Department of Physics, Al-Baha University, Al-Baha, Saudi Arabia \\ ${ }^{3}$ Department of Physics, University of Calabria, Rende, Italy
}

Email address:

salih33715@gmail.com (S. M. Osman)

${ }^{*}$ Corresponding author

\section{To cite this article:}

Salih Mohamed Osman, Asma Mohamed Elhussein, Fatma Osman Mahmoud, Mohammed Bilal Sabahelkher, Lorenzo Caputi, Andrea Scarcello, Francesca Alessandro. Obtained Carbon Nano-onions from Underwater Arc Discharge Without the Complex Purification Procedures. American Journal of Nanosciences. Vol. 7, No. 1, 2021, pp. 23-27. doi: 10.11648/j.ajn.20210701.14

Received: January 12, 2021; Accepted: February 3, 2021; Published: February 23, 2021

\begin{abstract}
Carbon nano-onions (CNOs), which consist of concentric graphitic shells, currently attract much attention because of their unique structural and physical properties, which are different from the properties of the other carbon nanomaterials such as fullerenes, graphene, and carbon nanotubes (CNTs). Due to their small size, the large external surface area and high conductivity, CNOs are used for supercapacitor applications. The arc discharge underwater is an effective and simple method for the synthesis of larger CNOs in reasonable quantities. In this research, we have been obtained carbon nanomaterials using arc discharge in water between two high purity graphite electrodes. The main experimental techniques used to characterize carbon nanostructures have been Transmission Electron Microscopy (TEM) and Raman Spectroscopy. Among them, Raman spectroscopy is the most useful non-destructive technique capable of differentiating between these various structures. Our TEM images showed that the samples collected from the material floating on the water surface consist CNOs with other carbon nanomaterials such as CNTs. We observed for the first time the formation of solid agglomerate on the cathode surface. Raman and TEM results revealed that the agglomerate is made exclusively of CNOs. The defragmentation of such agglomerate allows to obtain CNOs free of other carbon nanomaterials without the complex purification procedures needed for floating nanomaterials.
\end{abstract}

Keywords: CNOs, Graphite, Arc Discharge, Nanomaterials, TEM, Raman

\section{Introduction}

The arc discharge method offers an alternative to vacuum processes for the synthesis of larger CNOs in reasonable quantities. In addition, the arc discharge method is considerably cheaper and simpler than other methods. CNOs obtained by this method exhibits diameters between 15 and $25 \mathrm{~nm}$ (20-30 shells). In 2001 Sano and co-workers reported the arc discharge synthesis of CNOs underwater $[1,2]$. In this method, two high purity graphite rods were electrically arced under water by applying a bias potential of 16-17 V between them and maintaining a constant current of $30 \mathrm{~A}$. During the synthesis, CNOs were obtained as a floating powder on the surface of the water while other subproducts such as multiwalled CNTs precipitated to the bottom of the container. Xu and co-workers reported later that the mean CNOs size is 40 $\mathrm{nm}$, considerably larger than CNOs obtained from nanodiamonds [3].

Although CNOs obtained from arcing have not been studied as extensively as the ones obtained from annealing nanodiamonds some of their properties are known. The Raman Spectroscopy of these CNOs shows the D $\left(1344 \mathrm{~cm}^{-1}\right)$ and the $\mathrm{G}$ band (1569-1577 $\mathrm{cm}^{-1}$ ), respectively. The intensities ratio of the $D$ and $G$ peaks is used to estimate the degree of perfection of graphene planes. For example, the absence of the D peak in HOPG implies perfect graphene planes. Thus, a very weak D 
peak and a strong G peak from CNOs signify that only slight imperfections are present in the graphene planes of the onions [4-6]. There are many methods of synthesis of CNOs have appeared in the literature.

The experimental techniques used to characterize carbon nanostructures are TEM and Raman Spectroscopy. The previous researches devoted to study CNOs by Raman data [7-9] showed that contrary to what can be thought, their spectra are very different with respect to other CNPs such as fullerenes. Certainly, they resemble the spectra of graphene and graphite. This fact can be explained considering that the Raman spectra of fullerenes are dominated by the radial breathing mode and the so-called pinch pentagonal mode, linked with the vibrations of the atoms located in the vertices of pentagons in order to close the structure [10]. In CNOs, as the number of shells increases, the number of pentagons, relative to the number of hexagons, decreases, and then the contribution of the pentagonal modes becomes smaller. At the same time, the existence of several shells, in interaction through the Van der Waals forces, provokes the disappearance of the radial mode. Eventually, the vibration modes of multi-shell CNOs resemble those observed in curved graphene. The most important characteristic of the Raman spectra of CNOs is the shift at higher energies of the first-order Raman-allowed mode ( $\mathrm{G}$ band) due to the strain produced in the graphene planes by this curvature $[8,11]$.

It is important to remark that most of the information about Raman spectra of closed-shell carbon nanostructures refers to spherical nano-onions. In a work of Bogdanov et al. [11], there is an extensive Raman study of nano-onions produced by annealing of nano diamonds. In his work, Bogdanov includes not only the spherical CNOs, but also the polyhedral variants of these nanostructures obtained at high annealing temperatures. However, there is no distinction there, about the features of the Raman spectra depending on the kind of nano-onion.

As a matter of fact, the importance of polyhedral CNOs has been increasing in the last years. They are the kind of nanomaterial obtained in several experimental methods of preparation of CNOs, as for example, the one based on a submerged arc discharge of graphite electrodes in water [2, 7], a technique used to obtain CNOs in the present work and a method frequently utilized for producing high-purity nanomaterials [7, 12]. Codorniu Pujals et al obtained a typical Raman spectrum of the samples using underwater arc discharge method. The TEM image in the inset shows the polyhedral shape of CNOs [13].

\section{Experiment and Synthesis Procedure}

We created a metallic structure, consisting of two stainless steel flanges held together by three threaded columns to obtain a chassis. The upper plate was drilled in the center to allow assembly of the micrometer that would allow a good accuracy of advancement of the anode towards the cathode. The lower plate, instead, is characterized by a ceramic zone which isolates it electrically from the rest of the chassis; the cathode stand, made of copper, was precisely mounted on this area.

A power supply was used, that allowed the achievement of current values between $0-70 \mathrm{~A}$, with a difference of potential between $0-24 \mathrm{~V}$; this is controlled by a toroidal VARIAC variable power transformer. The AC exiting the power supply and regulated by the transformer, then converted into DC power by a rectifier bridge

Two graphite electrodes of similar purity of $99.99 \%$ (anode and cathode, 5 and $10 \mathrm{~mm}$ diameter respectively) cut to measure, were mounted on the chassis. Then we proceeded with the insertion in deionized water with a specific resistivity of $1.4 \mathrm{M} \Omega$ with a gap of $1 \mathrm{~mm}$. The arc discharge was initiated by contacting the anode with the cathode, the discharge voltage was $15 \mathrm{~V}$.

The arc discharge in water was stable maintained anodecathode gap of about $1 \mathrm{~mm}$. The discharge can be characterized as an anodic arc as the smaller anode electrode is consumed. The discharge proceeds until the almost total consumption of the anode, maintaining the synthesis chamber temperature under control: in our case, starting from a temperature equal to the ambient temperature $\left(\mathrm{T}=25^{\circ} \mathrm{C}\right)$ after approximately 70 seconds, a temperature of $83^{\circ} \mathrm{C}$ was reached, which was kept constant until the end of the discharge.

\section{Results and Discussions}

During the discharge, in the plasmatic zone area on the cathode, a gradual deposition of material occurred, thus forming a small disk-shaped tablet. In fact, it grew in correspondence of the center of the arc, and was in the maximum temperature zone. This assessment is essential to compare our results with those reported in the literature [14-16].

After the discharge, the synthesis chamber presented carbon nanoparticle (CNP) clusters scattered on the bottom and on the surface of the reaction water.

1. An intense grey powder is present on the surface, formed by floating clusters of carbon nanomaterials.

2. Instead, on the bottom, there are darker agglomerations.

3. The discoid presents a black colour, of a diameter of approximately $4 \mathrm{~mm}$, which was detached through the application of a weak force, leaving the cathode surface perfectly smooth.

Three sample groups were individuated from the synthesis chamber: powder floating on the water surface (S), the material precipitated on the bottom of water (B), and Discoid formed on cathode (D).

The surface $(\mathrm{Sp})$ and bottom $(\mathrm{Bp})$ particles were sonicated for $3.5 \mathrm{~h}$, in order to obtain a better dispersion of the carbonaceous material. After sonication, 2 phases of the material are presented: supernatant and precipitate; the precipitate material was heated to $80^{\circ} \mathrm{C}$ to evaporate water, thus the $\mathrm{Sp}$ and Bp samples were obtained.

The discoid sample (Ds) was divided to two pieces, one heated to $400{ }^{\circ} \mathrm{C}$ and the other without heating, then they have been converted to powder using a grinding machine, 
and the resulting powder materials of Ds were collected.

The samples of (Sp), (Bp) and (Ds) were characterised by microscopic and spectroscopic techniques. TEM was performed in JEOL Jem-2100, and Raman spectroscopy was made by using a Horiba-Jobin-Ivon Labram Raman spectrometer with a $\mathrm{He} \mathrm{Ne}$ laser $(632.8 \mathrm{~nm})$ as excitation source.

TEM images reveal that the samples $\mathrm{Sp}$ and $\mathrm{Bp}$ are conformed by same CNPs, although were obtained from different zones of synthesis chamber clearly showing the presence of CNOs and CNTs of various dimensions, and also some thin structures. Figure 1 shows agglomerates of CNTs with CNOs surrounding. The CNOs present dimensions of the order of tens of nanometers and CNTs with diameters of the order of some nanometers.

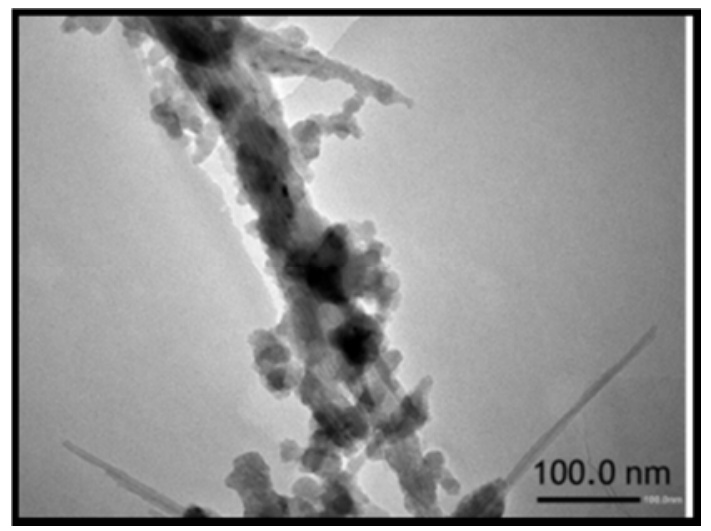

Figure 1. TEM image shows clearly agglomerates formed only of CNOs and CNTS.

Moreover, other image reveals also the presence of some larger particles with order dimensions of hundreds of nanometers. Figure 2 evidences a $\mathrm{CNO}$ of high dimension $640 \mathrm{~nm}$, and its diffraction image acquired by select-area electron diffraction (SAED). It shows a pattern that can be attributed to a hexagonal structure with rotational disorder.

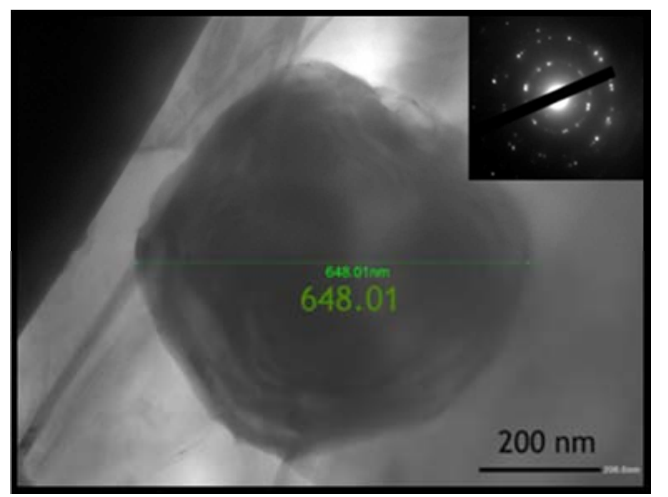

Figure 2. TEM image of a CNO of high dimension and inset SEAD image.

The Raman spectrum from Sp samples is indistinguishable of the spectrum from Bp sample, and this is indicative of the fact that they are constituted by very similar carbon structures; therefore, we analyzed one Raman spectrum as representative for both samples.
The similarity between Raman spectra of Sp and graphite shown in Figure 3 suggests that the anode fragments play a decisive role in determining the shape of Raman spectra of Sp. Furthermore, such fragments show a structural disorder (defects, reduced flake dimensions) that is increased compared to the original material, as shown by the increased intensity of the D peak.

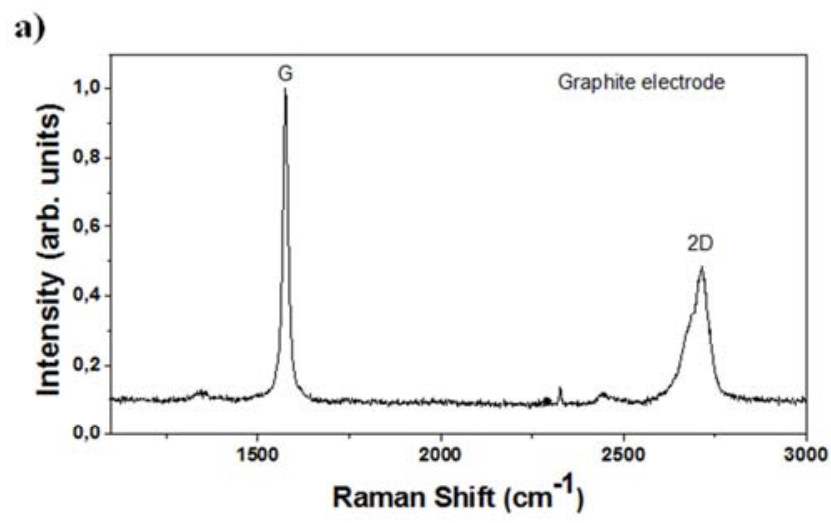

b)

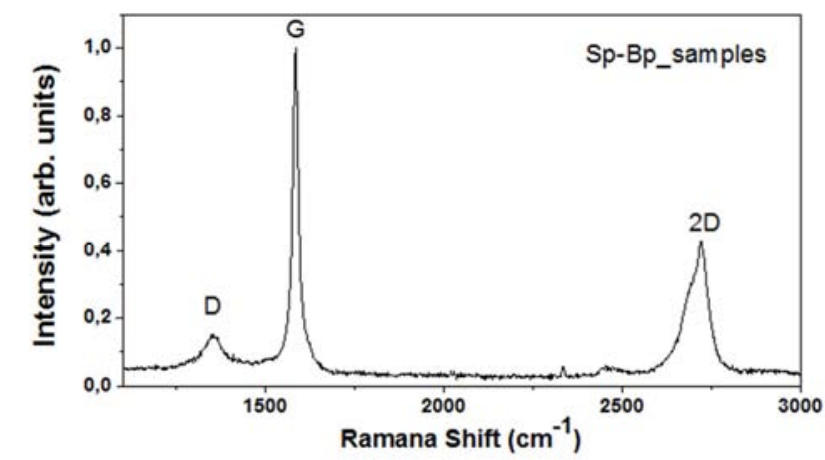

Figure 3. a) Raman spectra of graphite and b) floating CNPs formed on surface (lower).

Different results respect to Discoid sample (Ds) are observed on samples obtained by the grinded dust of the solid discoid fragmentation formed in the plasmatic zone. The TEM characterization of Ds sample (Figure 4), shows the presence of fragments consists a large proportion of CNOs with dimensions estimated at tens of nanometers. In contrast, the CNTs have become more reduction comparing with previous TEM results of $\mathrm{Sp}$ and $\mathrm{Bp}$. a)

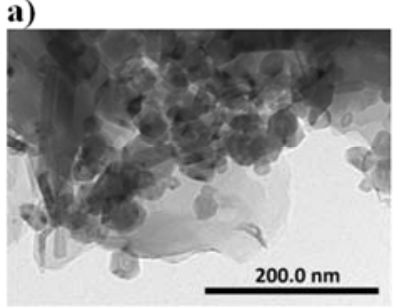

b)

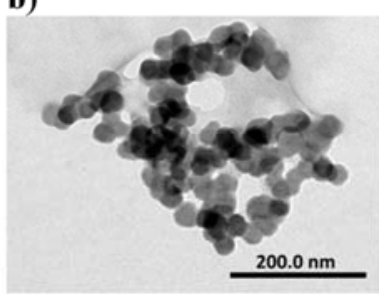

Figure 4. a) TEM image shows agglomerates of different CNPS (with more amounts of $\mathrm{CNOs}$ ). b) Image shows clearly agglomerates formed only of CNOS. 
The brilliant results have been acquired when we heated a part of the discoid fragment at $400^{\circ} \mathrm{C}$, and then grinded to obtain fine powder of the CNPs. The TEM characterization of this sample (Figure 5), shows the presence of fragments consisting exclusively of CNOs. More precisely, we have been observed in some fragments a polyhedral structure of CNOs with dimensions estimated at tens of nanometers.

a)

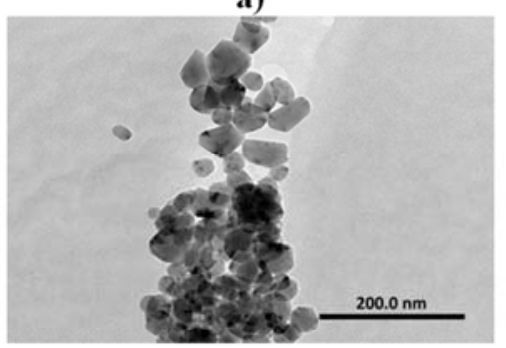

b)
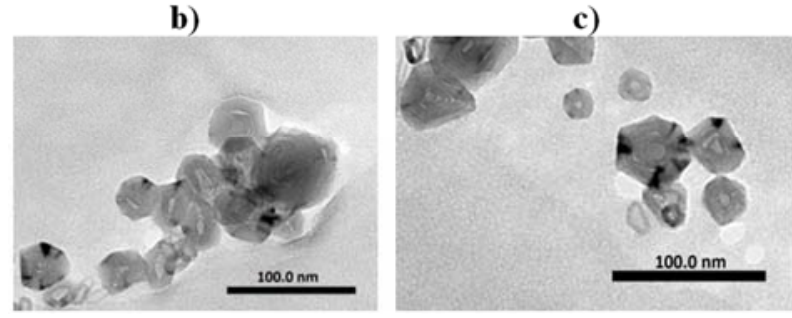

Figure 5. TEM images of grinded Ds sample. a) Fragment consisting of only CNOs clearly distinguishes each one. b) Image shows clearly polyhedral structures of CNOs, c) A polyhedral CNOs with dimensions of (20-50) $\mathrm{nm}$.

This is a completely new finding, showing that the discoid formed in the plasmatic zone only needs to be grinded to get a nanomaterial made only of CNOs, without the need of purification methods, as it would be necessary to obtain CNOs from the nanoparticles found on the surface or at the bottom of the vessel.

In Figure 6, we show the Raman spectra of Sp and Ds. The degree of structural disorder on graphitic planes present in the two samples is similar, as shown by the similar intensity of the D peaks. The most significant difference is found in the form of the 2D peak. Unlike the 2D peak of Sp sample, which presents the typical bimodal shape as in graphite, the spectrum obtained on Ds sample is essentially monomodal.

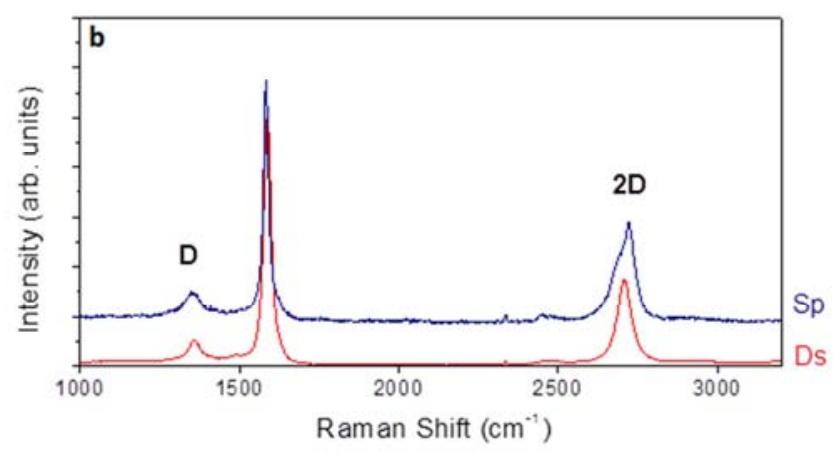

Figure 6. Plots of Raman spectra of material: (Red) discoid formed on the cathode (Ds), (Blue) floating on surface of water (Sp) compared with material of discoid formed on the cathode (Ds).
A monomodal 2D peak can be attributed to turbostratic graphite, namely graphite in which there is rotational disorder in the "stacking" between adjacent graphene sheets. As for the CNOs, it can be hypothesized that the planar areas are formed by turbostratic graphite, and that the curved parts, if present, do not significantly contribute to the $2 \mathrm{D}$ peak $[8,17]$. Figure 7 shows the fit of the 2D band evidence in Raman spectra (a) fit of 2D band of Ds sample with composition of polyhedral CNOs, and (b) the form of the 2D band reported by Codorniu Pujals et al. [10], obtained on polyhedral nano-onions in the presence of curved zones (such as fullerene). It has to be noted that the difference in the $2 \mathrm{D}$ peak location compared to our spectrum is due to the use of a laser with a different wavelength (632 nm instead of $514 \mathrm{~nm})$. a)

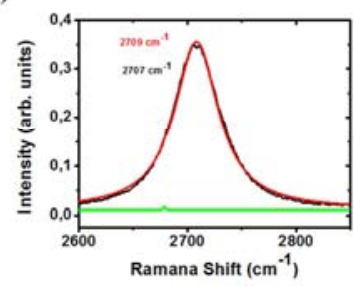

b)

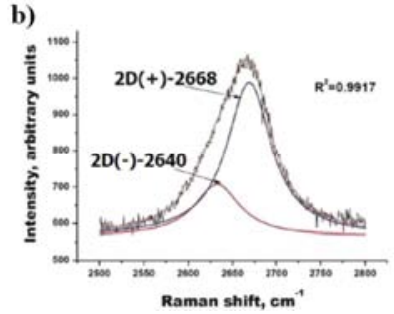

Figure 7. a) Fit of $2 D$ band Raman spectra Ds sample formed exclusively of CNOs fragments. b) Fit of the 2D band of polyhedral CNOs [13].

\section{Conclusions}

The results obtained show that the discharge procedure in deionized water between two graphite electrodes generated carbonaceous nanostructures with different geometry. In the samples collected at the bottom and on the surface of the reaction vessel, there are carbonaceous structures that do not have a particular symmetry. Perhaps the more important observation in our work is the formation of a solid agglomerate in the area of the plasma, which remains on the cathode but is not bonded to it. Furthermore, the TEM and Raman results show that there are notable differences between the particles deposited on the cathode forming the discoid and those present in suspension.

Finally, we confirm that this finding is very important because the material in the discoid which formed in the plasmatic zone does not need complex purification procedures to obtain CNOs free of the other carbonaceous materials as those needed for floating nanomaterials.

\section{References}

[1] N. Sano, H. Wang, M. Chhowalla, I. Alexandrou, and G. A. J. Amaratunga, "Synthesis of carbon 'onions' in water," Nature, vol. 414, no. 6863, p. 506, 2001.

[2] N. Sano, H. Wang, I. Alexandrou, M. Chhowalla, K. B. K. Teo and G. A. J. Amaratunga,"Properties of carbon onions produced by an arc discharge in water," J. Appl. Phys., vol. 92, no. 5, pp. 2783-2788, 2002.

[3] J. Guo, X. Wang, Y. Yao, X. Yang, X. Liu, and B. Xu, "Structure of nanocarbons prepared by arc discharge in water," Mater. Chem. Phys., vol. 105, no. 2-3, pp. 175-178, 2007. 
[4] D. Roy, M. Chhowalla, H. Wang, N. Sano, I. Alexandrou, T. W Clyne and G. A. J. Amaratunga, "Characterisation of carbon nano-onions using Raman spectroscopy," Chem. Phys. Lett., vol. 373 , no. $1-2$, pp. 52-56, 2003.

[5] Jiang-Bin Wu, ab Miao-Ling Lin, ab Xin Cong, ab He-Nan Liua and Ping-Heng Tan, Raman spectroscopy of graphenebased materials and its applications in related devices, Chem. Soc. Rev., 47, 1822, 2018.

[6] Elena F. Sheka, Yevgeny A. Golubev and Nadezhda A. Popova, Graphene Domain Signature of Raman Spectra of $\mathrm{sp}^{2}$ Amorphous Carbons, Nanomaterials, 10, 2020.

[7] F. Alessandro, A. Scarcello, M. D. Basantes Valverde, D C Coello Fiallos, S. M. Osman, A. Cupolillo, M. Arias, O. Arias de Fuentes, G. De Luca, A. Aloise, E. Curcio, G. Nicotra, C. Spinella and L. S. Caputi, Selective synthesis of turbostratic polyhedral carbon nano-onions by arc discharge in water, Nanotechnology 29, 325601, 10 pp, 0957-4484, 2018.

[8] W. S. Bacsa, W. A. de Heer, D. Ugarte, and A. Ch??telain, "Raman spectroscopy of closed-shell carbon particles," Chem. Phys. Lett., vol. 211, no. 4-5, pp. 346-352, 1993.

[9] E. D. Obraztsova, M. F. Workspace. c, S. Hayashi, V. L. Kuznetsov, Y. V. Butenko, and A. L. Chuvilin, "Raman identification of onion-like carbon," Carbon N. Y., vol. 36, no. 5-6, pp. 821-826, 1998.

[10] M. Dresselhaus, G. Dresselhaus, and P. Eklund, Science of fullerenes and Carbon Nanotubes. London: Academy Press, 965, ISBN: 9780080540771, 1996.
[11] K. Bogdanov, A. Fedorov, V. Osipov, T. Enoki, K. Takai, T. Hayashi, V. Ermakov, S. Moshkalev, A. Baranov, "Annealinginduced structural changes of carbon onions: High-resolution transmission electron microscopy and Raman studies," Carbon N. Y., vol. 73, pp. 78-86, 2014.

[12] R. Borgohain, J. Yang, J. P. Selegue, and D. Y. Kim, "Controlled synthesis, efficient purification, and electrochemical characterization of arc-discharge carbon nano-onions," Carbon N. Y., vol. 66, pp. 272-284, 2014.

[13] D. Codorniu Pujals, O. Arias de Fuentes, L. F. Desdin Garcia, E. Cazzanelli, and L. S. Caputi, "Raman spectroscopy of polyhedral carbon nano-onions," Appl. Phys. A Mater. Sci. Process., vol. 120, no. 4, pp. 1339-1345, 2015.

[14] I. Alexandrou, H. Wang, N. Sano, and G. A. J. Amaratunga, "Structure of carbon onions and nanotubes formed by arc in liquids," J. Chem. Phys., vol. 120, no. 2, pp. 1055-1058, 2004.

[15] D. Ugarte, "Curling and closure of graphitic networks under electron-beam irradiation.," Nature, vol. 359, no. 6397, pp. 707-709, 1992.

[16] N. Sano, T. Charinpanitkul, T. Kanki, and W. Tanthapanichakoon, "Controlled synthesis of carbon nanoparticles by arc in water method with forced convective jet," J. Appl. Phys., vol. 96, no. 1, pp. 645-649, 2004.

[17] Y. I. Kim, E. Nishikawa, and T. Kioka, "Carbon Nano Materials Produced by Using Arc Discharge in Foam," $J . K$. Phy. Soc, vol. 54, no. 3, pp. 1032-1035, 2009. 